\title{
Evaluation of Anthocyanin Stability in Surfactant Formulation from Extrudate Purple Potato (Solanum tuberosum L.)
}

\author{
Md Obyedul Kalam Azad, Young Seok Lim, Cheol Ho Park and Wie Soo Kang*
}

Department of Bio-Health Technology, College of Bio-Medical Science, Kangwon National University, Chuncheon 24341, Korea

\begin{abstract}
Anthocyanin has a strong antioxidant capacity but exhibit poor stability in water. Therefore, increasing stability of anthocyanin from purple potato (Solanum tuberosum L. var. love gold valley) was investigated in surfactant colloid followed by hot melt extrusion (HME). The hydrophilic Brij 35 and the lipophilic Span 80 were used to prepare association colloids of surfactant (ACS) and surfactant based purple potato extrudate (SPE). Result reveled that extraction of total phenol (TP), total flavonoid (TF), total anthocyanin (TA) and antioxidant activity (AA) was increased at 4, 2, 5 and 2 times, respectively, at 9 mM span 80 ACS compared to control (without surfactant). There was no significant reduction of anthocyanin was observed in SPE. Whereas, a significant reduction of anthocyanin was observed in control extrudate. The nano size particle $(<500 \mathrm{~nm})$ was achieved in Span 80 mediated ACS. Moreover, HME prepared $<300 \mathrm{~nm}$ particle in the SPE. The higher extraction of phenolic compound in ACS is may be due to chemical crosslink (adsorption and/or absorption) between surfactant micelle and anthocyanin molecule. It would be concluded that lipophilic association colloids can be used to protect anthocyanin from oxidation. However, further study needed to explain the mechanism and more understanding in this regard.
\end{abstract}

Keywords: Anthocyanin; Purple potato; Surfactant; Hot melt extrusion

\section{Introduction}

Purple potato (Solanum tuberosum L. var. love gold valley) is rich in anthocyanin and phenolic acids which have strong antioxidant capacities [1]. Dietary intake of anthocyanins reduces the risk of cancer, cardiovascular diseases, Alzheimer's and diabetes [2]. Moreover, anthocyanins are also used as natural colorants in food, beverage, cosmetics and paint industries [3]. However, the use of anthocyanin is limited because of major technological challenges since anthocyanin have low stability in aqueous medium due to their high heat sensitivity [4-6]. Several studies reported a logarithmic course of anthocyanin destruction with an arithmetic increase in temperature $[7,8]$.

In recent year extraction and separation of anthocyanin was investigated by the application of surfactant emulsion [9]. Studied reported that light, $\mathrm{pH}$, temperature, $\mathrm{NaCl}$, sugar, metal ion, and other factors affect anthocyanin solution stability [10,11]. Aditya et al. [12] prepared a surfactant emulsion to embed curcumin and catechin, and the results showed a remarkable enhancement of the stability of curcumin and catechin in a simulated gastrointestinal tract environment. McClements [13] reported that plant active compound can be incorporated into surfactant emulsion for longer stability without adversely affecting the food quality attributes.

Surfactant moieties form micelles in a solution above their critical micellar concentration (CMC). These micelles are composed of a hydrophilic head and lipophilic tail having capabilities of establishing chemical and physical crosslinked with both hydrophilic and lipophilic compounds $[14,15]$. Moreover, surfactant micelle alters the functional properties of biopolymers by binding with them or to displacing biopolymers from oil-water interfaces through competitive adsorption or modulating the crystallization in the given phases [16]. One of the most important aspects of surfactant is their ability to control stability and rheology of a food composition $[14,17,18]$.

The aim of the study was to determine the efficacy of the surfactant formulation as an encapsulating agent for the protection of anthocyanin compound. This proposed encapsulating design might improve the stability of anthocyanin in food processing industries.

\section{Materials and Methods}

\section{Flour preparations}

Purple potato was purchased from the Chuncheon, Korea local market. Samples were cut into slices (2-3 mm thickness) and freeze dried (Ilshin BioBasae, FD 5510S- FD 5520S, Korea). The freeze dried potato was blended using an electrical blender (Model No. Blixer 5 plus, Robot coup, USA) and prepared coarse powder. Potato powder was milled by a pin crusher (JIC-P10-2; Myungsung Machine, Seoul, Korea) equipped with a 30-mesh sieve. The milled powder was fractionated using a sieve shaker (CG-213, Ro-Top, Chunggye Industrial Mfg. Co., Seoul, Korea) equipped with a series of sieves (F $20 \mathrm{~cm}$ ). The powder was passed through $300 \mu \mathrm{m}$ mesh size sieves, and unpassed particles were grinded again with the pin crusher. Potato fine powders were then stored in a desiccator for the further use.

\section{Chemical and reagents}

Brij 35 (HLB: 16.9), Span 80 (HLB: 4.3), Phenolic reagent (Folin ciocalteu, $2 \mathrm{~N})$, Sodium bi-carbonate $\left(\mathrm{Na}_{2} \mathrm{CO}_{3}\right.$, Aluminum nitrate $\left(\mathrm{AlNO}_{3}\right)_{3}$, potassium acetate $\left(\mathrm{CH}_{3} \mathrm{CO}_{2} \mathrm{~K}\right)$, DPPH $(2,2$-diphenyl-1 picryl hydrazyl), were purchased from Sigma-Aldrich (St. Louis, MO, USA). All other chemicals used were of analytical grade and purchased from

*Corresponding author: Wie Soo Kang, Department of Bio-Health Technology, College of Bio-Medical Science, Kangwon National University Chuncheon 24341, Korea Tel: +82-033-250-6494; Fax: +82-033-250-6470; E-mail: kangwiso@kangwon.ac.kr

Received August 02, 2018; Accepted August 16, 2018; Published August 21, 2018

Citation: Kalam Azad MO, Lim YS, Park CH, Kang WS (2018) Evaluation of Anthocyanin Stability in Surfactant Formulation from Extrudate Purple Potato (Solanum tuberosum L.). J Food Process Technol 9: 750. doi: 10.4172/21577110.1000750

Copyright: () 2018 Kalam Azad MO, et al. This is an open-access article distributed under the terms of the Creative Commons Attribution License, which permits unrestricted use, distribution, and reproduction in any medium, provided the original author and source are credited. 
Merck Chemical Corp. (Darmstadt, Germany). Deionized, distilled water (EC value $<0.3 \mu \mathrm{S} . \mathrm{cm}^{-1}$ ) was used for sample preparation.

\section{Surfactant formulation and sample preparation}

Surfactant emulsions were prepared in water with different molar concentration followed by $3,5,7,9$, and $11 \mathrm{mM}$. Formulations were well mixed using electric stirrer (Ultra Torque, Model BDC1850) with high shear in order to breakdown the kinetic energy barrier to ensure as surfactant is adequately dispersed.

\section{Extraction of phenolic compound from the purple potato}

One gram of potato fine powder was added in $100 \mathrm{ml}$ of different surfactant formulation in $200 \mathrm{ml}$ conical flask. The sample was shaken at $150 \mathrm{rpm}$, using shaking incubator (SI-900RF, JEIO TECH, Korea) for one hour at room temperature. The sample was filtered through $125 \mathrm{~mm}$ filter paper (Advantech 5B Tokoyo Roshi Kaisha, Japan) and then extract was stored in refrigerator at $-20^{\circ} \mathrm{C}$ for phenolic compound analysis.

\section{Preparation of extrudate formulation and HME configuration}

The extrudate of purple potato was developed $9 \mathrm{Mm}$ of Brij 35 and Span 80 surfactant agent using an STS-25HS twin-screw HME (Hankook E.M. Ltd., Pyoungtaek, Korea). The extruder was equipped with a round-shaped die $(1 \mathrm{~mm})$ and was operated at a feeding rate of $40 \mathrm{~g} / \mathrm{min}, 150 \mathrm{rpm}$ with high shear. The temperature profile from the feeding zone to die was $80 / 100 / 100 / 80 / 70^{\circ} \mathrm{C}$. The potato extrudate was dried in an oven at $50^{\circ} \mathrm{C}$ and then ground for further analysis.

\section{Particle size analysis}

The potato powder (PP) and potato extrudate powder (PEP) $(0.5$ g) was suspended in $50 \mathrm{ml}$ of distilled water. The supernatant was separated by centrifugation at 3,000 rpm for $10 \mathrm{~min}$. The particle size of the supernatant was studied using a light-scattering spectrophotometer (ELS-Z1000; Otsuka Electronics, Tokyo, Japan) with three replications.

\section{Determination of total phenolic content}

The total phenolic contents (TP) were determined by the Folin Ciocalteu assay [19]. A sample aliquot of $200 \mu \mathrm{l}$ was added to $200 \mu \mathrm{l}$ $1 \mathrm{~N}$ phenol reagent. The solution was allowed to stand for $3 \mathrm{~min}$ for reaction. To continue reaction, $400 \mu$ of $\mathrm{Na}_{2} \mathrm{CO}_{3}(10 \%$ in water $\mathrm{v} / \mathrm{v})$ was added and vortexed. The prepared sample was then incubated for 1 hour at room temperature. The absorbance was measured at $725 \mathrm{~nm}$ using a spectrophotometer (UV-1800 240 V, Shimadzu Corporation, Kyoto, Japan). The TP was expressed as Gallic acid equivalents (GAE) in dry weight basis.

\section{Determination of total flavonoid content}

The total flavonoid content (TF) was determined according to Ghimeray et al. [20]. Briefly, an aliquot of $0.5 \mathrm{ml}$ of sample $(1 \mathrm{mg} /$ $\mathrm{ml}$ ) was mixed with $0.1 \mathrm{ml}$ of $10 \%$ aluminium nitrate and $0.1 \mathrm{ml}$ of potassium acetate $(1 \mathrm{M})$. The mixture was vortexed and incubated for 40 min. The total flavonoid was measured using spectrophotometer at 415 $\mathrm{nm}$. The TF was expressed as $\mu \mathrm{g} / \mathrm{g}$ quercetin equivalent in dry weight basis.

\section{Determination of total anthocyanin content}

The content of total anthocyanin (TA) was determined by the $\mathrm{pH}$ differential method. Each extract $(0.5 \mathrm{~mL}$ of $)$ was diluted with $2.5 \mathrm{~mL}$ of $0.025 \mathrm{M}$ potassium chloride buffer, $\mathrm{pH} 1.0$ and $0.4 \mathrm{M}$ sodium acetate buffer, $\mathrm{pH} 4.5$, separately. The diluted solutions were incubated at room temperature for $15 \mathrm{~min}$. The absorbance was taken at 520 and $700 \mathrm{~nm}$ against a blank cell filled with distilled water using spectrophotometer. The TA was calculated according to equation described by Giusti and Wrolstad [21].

\section{DPPH free radical scavenging activity}

The antioxidant activity was determined on the basis of the scavenging activity of the stable 2, 2-diphenyl-1 picryl hydrazyl (DPPH) free radical according to methods described by Braca et al. [22]. $1 \mathrm{ml}$ of extract was added to $3 \mathrm{ml}$ of DPPH. The mixture was shaken vigorously and left to stand at room temperature in the dark for 30 mins. The absorbance was measured at $517 \mathrm{~nm}$ using a spectrophotometer. The percent inhibition activities of the sample were calculated against a blank sample using the following equation: inhibition (\%)=(blank sample-extract sample/blank sample) $\times 100$.

\section{Statistical analysis}

All data were expressed as Mean \pm SD of triplicate measurements. The obtained results were compared among the different surfactants concentration and types using a paired $t$ test in order to observe the significance differences at the level of $5 \%$. The paired $t$-test between mean values was analyzed by MINITAB version 16.0 (Minitab Inc., State College, PA, USA).

\section{Results and Discussion}

Effect of surfactant on the particle size reduction of the extrudate and non extrudate potato powder

It is clearly observed that nanonization was enhanced of the extrudate solid by the hot melt extrusion process (Figure 1). The particle size of the non-extrudate sample was achieved at $5669 \mathrm{~nm}$, whereas the size was $1584 \mathrm{~nm}$ in the extrudate sample in control treatment. It is reported that HME extrusion is the most suitable process to enhance the amorphization of crystal materials by reducing particle size $[23,24]$. The particle size reduction stragety results in increased surface area, decreased diffusional distance, and increased dissolution rates [25-27].

Among the formulations, the nano particle size $(289 \mathrm{~nm})$ was achieved in the lipophilic Span 80 mediated extrudate compared to Brij 35 . The effect of the surfactant on the particle size, polydispersity, type of particle size distribution and structure of nanoparticles synthesized has been studied by Toton et al. [28]. It is also reported that surfactant emulsion produce a nanoparticle from a diverse variety of materials, including metals [29], silica [30], polymers [31] were studied. In previous experiment $\mathrm{CuSo}_{4}$ nano composite was prepared by the surfactant mediated HME process [32]. Similarly, in this study for the purple potato powder the nano size particle was achieved in Span 80

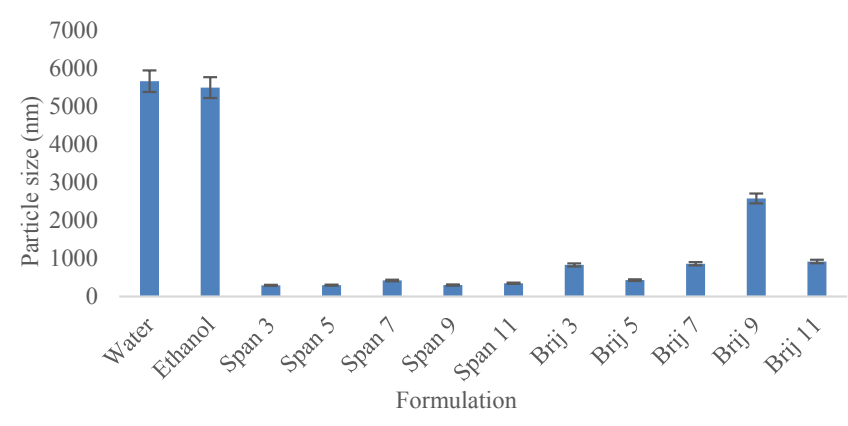

Figure 1: Effect of the surfactant concentration on the particle size distribution of the active compound from the purple potato. 
Citation: Kalam Azad MO, Lim YS, Park CH, Kang WS (2018) Evaluation of Anthocyanin Stability in Surfactant Formulation from Extrudate Purple Potato (Solanum tuberosum L.). J Food Process Technol 9: 750. doi: 10.4172/2157-7110.1000750

Page 3 of 5

agent. Further investigation is needed to clarify the mechanism behind this nanonization process.

\section{Effect of surfactant and solvent (water and ethanol) on the extraction of the phenolic compound}

It is clearly shown from Table 1 that surfactant efficiently extracted phenolic compound from the purple potato compared to water and ethanol. An associate colloidal of the dispersed active compound was prepared by the surfactant agent. The phenolic compound of the Purple potato was dispersed in water and ethanol. The content of the total phenol $(1053,1952 \mu \mathrm{g} / \mathrm{g})$, total flavonoid $(580,721 \mu \mathrm{g} / \mathrm{g})$, total anthocyanin $(150,287 \mu \mathrm{g} / \mathrm{g})$ and antioxidant activity $(42 \%, 57 \%)$, respectively in water and ethanol solvent (Table 1). It is attributed that polar solvents (water and ethanol) extracted only polar components, but non-polar and other protein binding matrix of active compounds were not extracted, thus least extraction was achieved in conventional solvents.
The highest active compound was extracted at the $9 \mathrm{mM}$ concentration which is selected as a critical micelle concentration (CMC) of the purple potato. Total phenolic compound (PC), total flavonoid (TF), total anthocyanin (TA) and antioxidant activity (AA) was increased 4, 2, 4 and 2 times, respectively at $9 \mathrm{mM}$ concentration compared to water extraction. Lipophilic surfactant Span 80 showed highest efficiency to extract PC, TF, TA and AA compared to Brij 35 at the same concentration. It is observed that extraction efficiency for active compounds from purple potato linearly increased with increasing surfactant concentration till $9 \mathrm{mM}$. It is shown that total phenolic compound (PC), total flavonoid (TF), total anthocyanin (TA) and antioxidant activity (AA) was increased 4, 2, 4 and 2 times, respectively at $9 \mathrm{mM}$ concentration compared to water extraction.

The maximum extraction efficiency has been obtained at $9 \mathrm{mM}$ concentration and further increased in surfactant concentration showed declined trends in extraction efficiency. Surfactant concentration more than their CMC level makes an abrupt change in physicochemical

\begin{tabular}{|c|c|c|c|c|c|c|c|c|c|c|c|c|}
\hline \multirow{3}{*}{ Phenol compo-und } & \multirow{3}{*}{ Water } & \multirow{3}{*}{ EtoH } & \multicolumn{10}{|c|}{ Concentration (mM) } \\
\hline & & & \multicolumn{2}{|c|}{3} & \multicolumn{2}{|c|}{5} & \multicolumn{2}{|c|}{7} & \multicolumn{2}{|c|}{9} & \multicolumn{2}{|c|}{11} \\
\hline & & & Span 80 & Brij 35 & Span 80 & Brij 35 & Span 80 & Brij 35 & Span 80 & Brij 35 & Span 80 & Brij 35 \\
\hline TPC & $1053 e$ & $1952 c$ & $1608 d$ & $1207 e$ & $2343 b$ & $1121 \mathrm{e}$ & $2516 b$ & 945 & $4546 a$ & $1911 c$ & $4241 a$ & $1830 c$ \\
\hline TF & $580 d$ & $721 \mathrm{c}$ & $703 c$ & 511 & $745 c$ & $545 d$ & $554 d$ & $473 d$ & $1120 a$ & $561 d$ & $1006 b$ & $508 d$ \\
\hline TA & $150 \mathrm{e}$ & $267 d$ & $301 \mathrm{c}$ & $210 c$ & $380 c$ & $312 c$ & $415 c$ & $380 c$ & $618 a$ & $401 \mathrm{c}$ & $520 b$ & $310 c$ \\
\hline DPPH & $42 e$ & $57 c$ & $60 b$ & $39 d$ & $63 b$ & $48 d$ & $63 b$ & $45 d$ & $75 a$ & $54 \mathrm{c}$ & $51 \mathrm{c}$ & $41 d$ \\
\hline
\end{tabular}

Results shown are mean \pm SD $(n=3)$. Same letters within the row are not statistically difference at $p<0.05$.

Table 1: Effect of the surfactant concentration on the total phenol, flavonoid, anthocyanin and antioxidant activity of the purple potato.

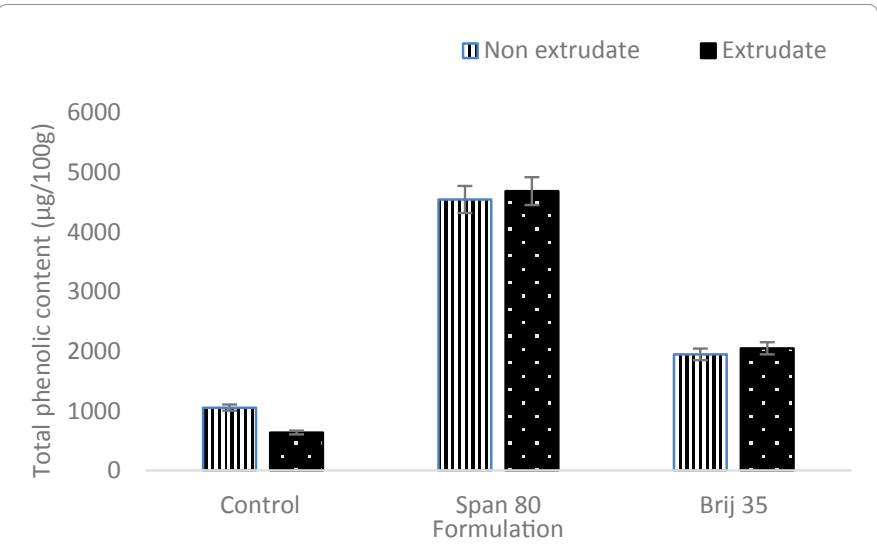

Figure 2: Effect of the surfactant concentration on the total phenolic compound from the purple potato.

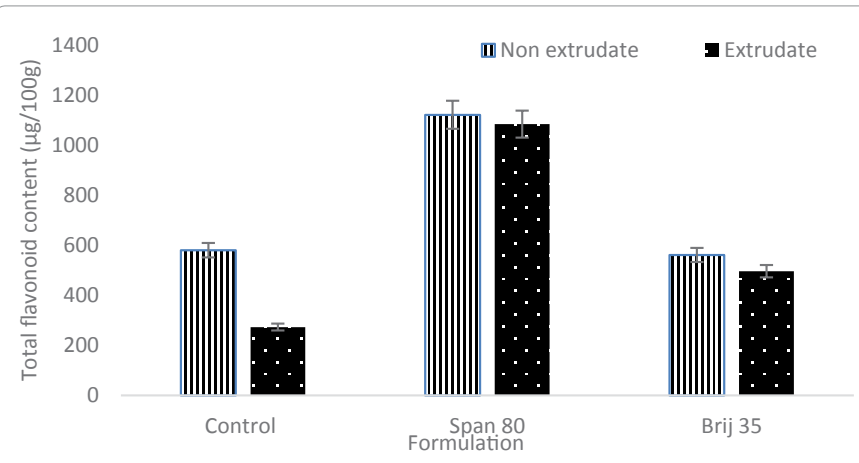

Figure 3: Effect of the surfactant concentration on the total flavonoid compound from the purple potato.

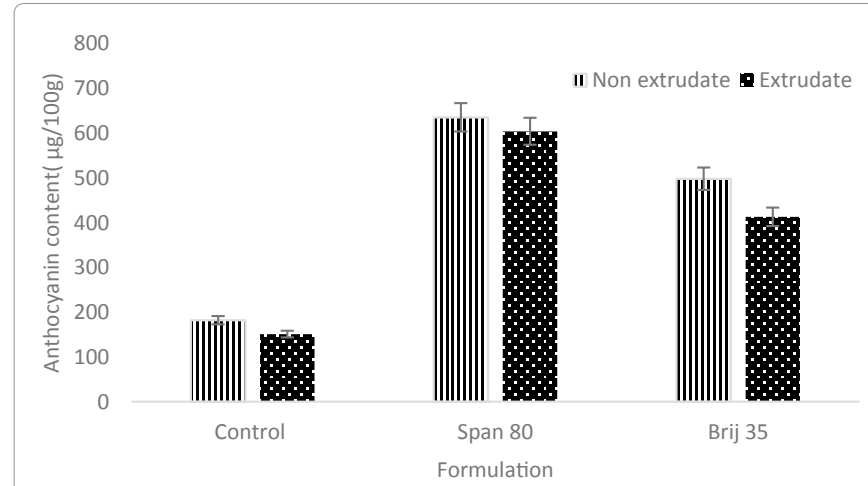

Figure 4: Effect of the surfactant concentration on the anthocyanin compound from the purple potato.

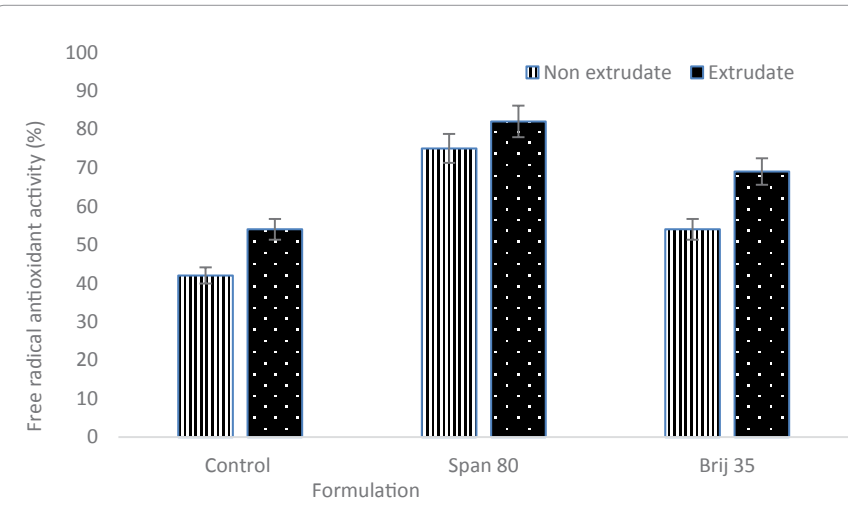

Figure 5: Effect of the surfactant concentration on the free radical antioxidant activity of the active compound from the purple potato. 


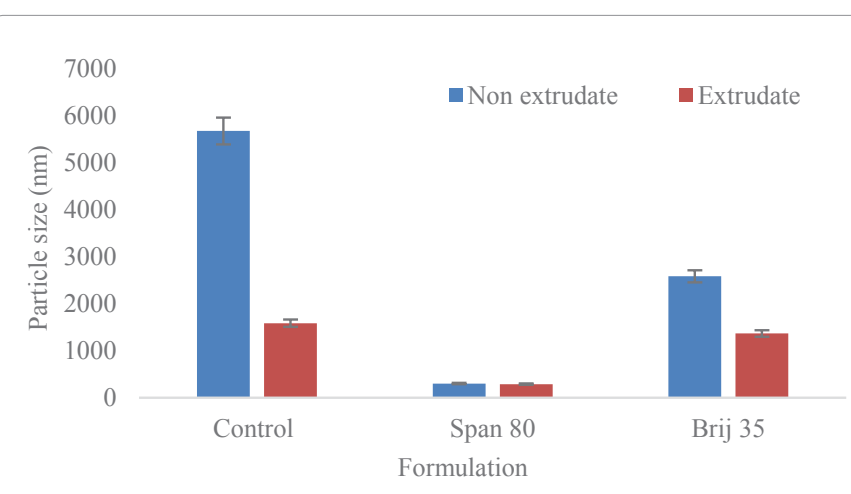

Figure 6: Effect of the surfactant concentration on the particle size of the active compound.

properties of surfactant such as surface tension, electrical conductivity and osmotic pressure. This is because monomers are amphiphilic and so are surface active whereas, micelles are hydrophilic and therefore have low surface activity [28]. Result demonstrated that lipophilic surfactant Span 80 showed highest efficiency in extracting TP, TF, TA, and AA compared to control and Brij 35 at $9 \mathrm{mM}$ concentration.

Surfactant based assemblies form associated colloidal which dissolve both polar and non-polar matrix of active compounds due to their amphiphilic nature [33]. Span 80 surfactant mediated aqueous solution established a chemical crosslinked with polar and non-polar molecules of polyphenol compounds and formed hydrogen bond between them, as a consequences, higher extraction was attained.

As the anthocyanin easily oxidized during extraction in Brij 35 mediated solution therefore TF contents were found lower than those achieved with Span 80 mediated assemblies. Studied showed that non-ionic hydrophilic surfactant Brij 35 effectively extracted active compounds from apple juice compared to lipophilic surfactant and conventional solvents $[33,34]$. However, in our study, Span 80 had an effective extracting moiety having a capacity of anthocyanin protection from oxidation in purple potato. Appropriate surfactant for each particular application depending on the type of active ingredient to be encapsulated, the nature of food matrix and functional attributes [13].

\section{Effect of melt extrusion on the extraction of the active compound}

Total phenolic, total flavonoid and anthocyanin was quantified from the extrudate purple potato (Figures 2-6). Result demonstrated that there were no significant changes of the content of the total phenol, total flavonoid and total anthocyanin in extrudate solid compared to non exturdate. The possible mechanism involved in this processes is the developing coating layer around the active compound in the aqueous media by the surfactant micelle. The chemical formula of Brij $35\left[\mathrm{C}_{12} \mathrm{H}_{25}\left(\mathrm{OCH}_{2} \mathrm{CH}_{2}\right)_{23} \mathrm{OH}\right]$ and Span80 $\left[\left(\mathrm{C}_{24} \mathrm{H}_{44}\right)\right.$ O6] exposed that span 80 is more organic polar in nature, having a longer hydrophilic tail and a shorter hydrophobic head in which hydrophilic flavonoid are dispersed in span 80 mediated colloidal system. Micelles head of span 80 make a hydrophobic coating over anthocyanin molecule in a colloidal emulsion. This coating encapsulate active compound especially anthocyanin during processing thus a constant phenolic compound was achieved [35]. The lipophilic surfactant micelle created a hydrophilic core surrounded by lipophilic layer. The active compound including anthocyanins are protected by their strong hydrophilic core and lipophilic layer, respectively. Koo et al. [32] developed a CuSO4 nano composite using surfactant agent and showed an enhanced pharmacokinetics activity in vivo. It proves the encapsulating capacity of surfactant for the metal ion.

\section{Conclusion}

The lipophilic surfactant Span 80 showed higher capabilities to protect anthocyanin from oxidation during processing compared to hydrophilic Brij 35. There was no significant reduction of active compound observed in extrudate solid compared to non extrudate purple potato powder. This finding would help for better understanding to develop a processing technology to enhance anthocyanin stability from the purple potato.

\section{Acknowledgment}

This research was supported by the Kangwon National University research grant (Grant No. C1011720-01-01)

\section{References}

1. Nayak B, Berrios DJ, Joseph R, Juming T (2011) Effect of extrusion on the antioxidant capacity and color attributes of expanded extrudates prepared from purple potato and yellow pea flour mixes. J Food Sci 76: 874-883.

2. Andersen OM, Jordheim M (2006) The anthocyanins: Flavonoids-Chemistry, Biochemistry and Applications. CRC Press, Boca Raton, USA.

3. Castaneda-Ovando $A$, Pacheco-Hernández MDL, Páez-Hernández ME Rodríguez JA, Galán-Vidal CA (2009) Chemical studies of anthocyanins: a review. Food Chem 113:859-871.

4. Fennema RO (2008) Food chemistry. (3rd edtn), Boca Raton, London.

5. Routray W, Orsat V (2011) Blueberries and their anthocyanins: Factors affecting biosynthesis and properties. Compr Rev Food Sci Food Saf 10: 303-320.

6. Clifford MN (2000) Anthocyanins-Nature, occurrence and dietary burden. J Sci Food Agric 80: 1063-1072.

7. Drdak M, Daucik $P$ (1990) Changes of elderberry (Sambucus nigra) pigments during the production of pigment concentrates. Acta Aliment 19: 3e7.

8. Rhim, JW (2002) Kinetics of thermal degradation of anthocyanin pigment solutions driven from red flower cabbage. Food Sci Biotechnol 11: 361e364.

9. Chen J, Dickinson E (1998) Viscoelastic Properties of Protein-Stabilized Emulsions: Effect of Protein-Surfactant Interactions. J Agric Food Chem 46: 91-97.

10. Tiwari BK, O'Donnell CP, Patras A, Cullen PJ (2008) Anthocyanin and ascorbic acid degradation in sonicated strawberry juice. J Agric Food Chem 56: 10071 10077.

11. Yang $Y$, Yuan $X H, X u Y Q, Y u Z Y(2015)$ Purification of Anthocyanins from Extracts of Red Raspberry Using Macroporous Resin. Int J Food Prop18: 10461058.

12. Aditya NP, Macedo AS, Doktorovov S, Souto EB, Kim S, et al. (2014) Development and Evaluation of Lipid Nanocarriers for Quercetin Delivery: A Comparative Study of Solid Lipid Nanoparticles (SLN), Nanostructured Lipid Carriers (NLC), and Lipid Nanoemulsions (LNE). LWT-Food Sci Technol 59: $115-121$.

13. McClements DJ (2011) Edible nano emulsions: Fabrication, properties, and functional performance. Soft Matter 7: 2297-2316.

14. McClements DJ, Xiao H (2012) Potential biological fate of ingested nanoemulsions: Influence of particle characteristics. Food Funct 3: 202-220.

15. Berthod A, Garcia-Alvarez-Coque MC (2000) Micellar liquid chromatography Chromatographic science series. (1st edtn), New York: Marcel Dekker, Inc.

16. Bordbar AK, Hosseinzadeh R (2006) Binding of cetylpyridinium chloride to glucose oxidase. Colloids Surf B Biointerfaces 53: 288-295.

17. Kralova I, Sjoblom J (2009) Surfactants used in food industry: A review. J Dispersion Sci Tech 30: 1363-1383.

18. Rosen MJ (2004) Surfactants and interfacial phenomena. (3rd edtn), Hoboken: John Wiley and Sons Inc. 
Citation: Kalam Azad MO, Lim YS, Park CH, Kang WS (2018) Evaluation of Anthocyanin Stability in Surfactant Formulation from Extrudate Purple Potato (Solanum tuberosum L.). J Food Process Technol 9: 750. doi: 10.4172/2157-7110.1000750

19. Singleton VL, Rossi JA(1965) Colorimetry of total phenolics with phosphomolybdicphosphotungstic acid reagents. Am J Enology Vitic 16: 144-458.

20. Ghimeray AK, Sharma P, Phoutaxay P, Salitxay T, Woo SH, et al. (2014) Far infrared irradiation alters total polyphenol, total flavonoid, antioxidant property and quercetin production in Tartary buckwheat sprout powder. J Cereal Sci 59 : 167-172.

21. Giusti MM, Wrolstad RE (2001) Characterization and measurement of anthocyanins by UV-Visible spectroscopy. Curr Prot Food Anal Chem.

22. Braca A, Fico G, Morelli I, Simone F, Tome F, et al. (2003) Antioxidant and free radical scavenging activity of flavonol glycosides from different Aconitum species. J Ethnopharmacol 86: 663-678

23. Lee SY, Lee JJ, Nam SY, Kang WS, Yoon IS, et al. (2017) Fabrication of polymer matrix-free nanocomposites based on Angelica gigas Nakai extract and their application to breast cancer therapy. Colloid Surf B: Biointerfaces 159:781-790.

24. Maniruzzaman M, Rana MM, Boateng JS, Motchell JC, Douroumis D (2012) Dissolution enhancement of poorly water-soluble APIs processed by hot-melt extrusion using hydrophilic polymers. Drug Develop Ind Pharm 39: 218-227.

25. Hu J, Johnston KP, Williams RO (2004) Nanoparticle engineering processes for enhancing the dissolution rates of poorly water soluble drugs. Drug Develop Ind Pharm 30: 233-245

26. Repka MA, Battu SK, Upadhye SB, Thumma S, Crowley MM, et al. (2007) Pharmaceutical applications of hot-melt extrusion: Part II. Drug Develop Ind Pharm 33: 1043-1057.

27. Merisko-Liversidge EM, Liversidge GG (2008) Drug nanoparticles: Formulating poorly water-soluble compounds. Toxic Pathol 36:43-48
28. Tonon RV, Brabet C, Hubinger MD (2010) Anthocyanin stability and antioxidant activity of spray dried açai (Euterpe oleracea Mart.) juice produced with different carrier agents. Food Res Int 43: 907-914.

29. Stubenrauch C, Wielpütz T, Sottmann T, Roychowdhury C, DiSalvo FJ (2008) Microemulsions as templates for the synthesis of metallic nanoparticles. Colloid Surf A 317: 328-338.

30. Arriagada FJ, Osseo-Asare K (1999) Synthesis of Nanosize Silica in a Nonionic Water-in-Oil Microemulsion: Effects of the Water/Surfactant Molar Ratio and Ammonia Concentration. J Colloid Interface Sci 211: 210-220.

31. Hammouda A, Gulik T, Pileni MP (1995) Synthesis of nanosize latexes by reverse micelle polymerization. Langmuir. 11: 3656-3659.

32. Koo JS, Lee SY, Nam S, Azad MOK, Kim M, et al. (2018) Preparation of cupric sulfate based self emulsifible nanocomposites and their application to the photothermal therapy of colon adenocarcinoma. J Biochem Biophys Res Commun.

33. Sharma S, Kori S, Parmar A (2015) Surfactant mediated extraction of total phenolic contents (TPC) and antioxidants from fruits juices. Food Chem 185: 284-288.

34. Hosseinzadeh R, Khorsandi K, Hemmaty S (2013) Study of the effect of surfactants on extraction and determination of polyphenolic compounds and antioxidant capacity of fruits extracts. PLoS one 8: E57353.

35. Akhtar M, Murray BS, Afeisume El, Khew SH (2014) Encapsulation of flavonoid in multiple emulsions using spinning disc reactor technology. Food Hydrocoll 34: $62-67$. 